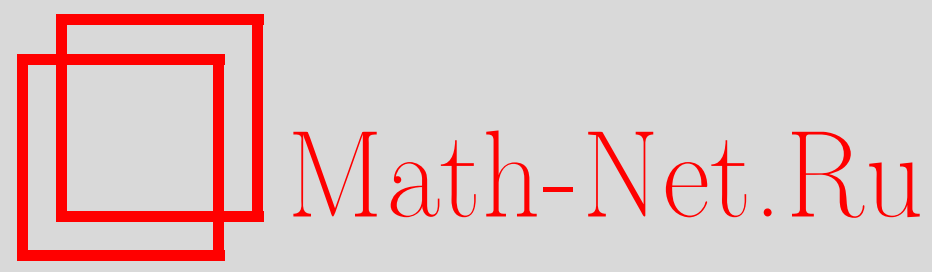

А. А. Толстоногов, Строго выставленные точки разложимых множеств в пространствах интегрируемых по Бохнеру функций, Матем. заметки, 2002, том 71, выпуск 2, 298-306

DOI: https://doi.org/10.4213/mzm348

Использование Общероссийского математического портала Math-Net.Ru подразумевает, что вы прочитали и согласны с пользовательским соглашением http://www.mathnet.ru/rus/agreement

Параметры загрузки:

IP : 44.207 .124 .84

26 апреля 2023 г., 06:04:43

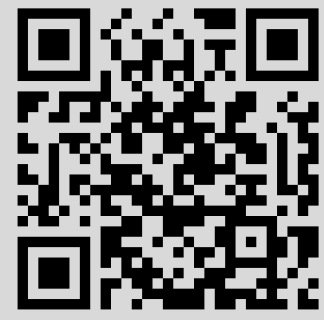




\title{
СТРОГО ВЫСТАВЛЕННЫЕ ТОЧКИ РАЗЛОЖИМЫХ МНОЖЕСТВ В ПРОСТРАНСТВАХ ИНТЕГРИРУЕМЫХ ПО БОХНЕРУ ФУНКЦИЙ
}

\section{А. А. Толстоногов}

\begin{abstract}
Дается характеризация строго выставленных точек выпуклого замкнутого ограниченного разложимого множества $\Gamma \subset L_{p}(T, X), 1 \leqslant p<\infty$, в терминах строго выставленных точек значений отображения $F: T \rightarrow 2^{X}$, которое является многозначным представлением множества $\Gamma$. Как следствие получены необходимые условия, характеризующие строго выставленные точки единичного шара из $L_{p}(T, X), 1 \leqslant p<\infty$, в терминах подобных точек единичного шара из $X$.
\end{abstract}

Библиографой: 16 названий.

Изучение характерных точек выпуклых замкнутых разложимых множеств является актуальной задачей в связи с использованием этих множеств в различных приложениях. Типичным примером разложимого множества является совокупность всех измеримых селекторов измеримого многозначного отображения с непустыми значениями. В приложениях, например в теории дифференциальных включений, теории оптимального управления и т.д., разложимые множества фигурируют как значения многозначного оператора Немьщкого (оператора суперпозиции).

Характеризация экстремальных (крайних) точек совокупности всех измеримых селекторов интегрально ограниченного многозначного отображения с вьпуклыми замкнутыми значениями изучалась в [1], а характеризация этих же точек вьпуклого замкнутого ограниченного разложимого множества в пространстве интегрируемых по Бохнеру функций рассматривалась в работе [2].

В недавно вьшедшей работе [3] изучались острые (denting) точки совокупности всех измеримых селекторов интегрального ограниченного многозначного отображения с выпуклыми замкнутыми значениями, а характеризация строго выставленных точек такого же множества для частного вида интегрально ограниченного многозначного отображения рассмотрена в работе [4].

В настоящей работе мы даем характеризацию строго выставленных точек замкнутых выпуклых ограниченных разложимых множеств в достаточно общих пространствах интегрируемых по Бохнеру функций. Наши результаты как следствие включают результаты работы [4], а их доказательство базируется на иных предпосылках.

Работа выполнена при частичной поддержке Российского фонда фундаментальных исследований, грант № 99-01-00216. 
1. Основные обозначения и предварительные сведения. В обозначениях и определениях мы следуем [5]. Пусть $(Y,\|\cdot\|)$ - банахово пространство, $\left(Y^{\prime},\|\cdot\|\right)$ - его топологически сопряженное пространство, $\left\langle y, y^{\prime}\right\rangle$ - каноническая билинейная форма, устанавливающая двойственность между $Y$ и $Y^{\prime}, Y_{\sigma}$ и $Y_{\sigma}^{\prime}-$ пространства $Y$ и $Y^{\prime}$ со слабыми $\sigma\left(Y, Y^{\prime}\right)$ и $\sigma\left(Y^{\prime}, Y\right)$ топологиями соответственно.

Пусть $D \subset Y$ - ограниченное множество и $y^{\prime} \in Y^{\prime}, y^{\prime} \neq 0$. Обозначим $M\left(D, y^{\prime}\right)=$ $\sup \left\{\left\langle y, y^{\prime}\right\rangle \mid y \in D\right\}$. Если $\alpha>0$, то множество

$$
S\left(D, y^{\prime}, \alpha\right)=\left\{y \in D \mid\left\langle y, y^{\prime}\right\rangle>M\left(D, y^{\prime}\right)-\alpha\right\}
$$

назьвается срезом (slice [5]) множества $D$.

Пусть $K \subset Y$ - вьпуклое замкнутое ограниченное множество с топологией, индуцированной топологией пространства $(Y,\|\cdot\|)$.

Точка $y_{*} \in K$ называется строго выставленной (strongly exposed [5]) точкой множества $K$, если существует элемент $y^{*} \in Y^{\prime}$ такой, что

a) $\left\langle y_{*}, y^{*}\right\rangle=\sup \left\{\left\langle y, y^{*}\right\rangle \mid y \in K\right\}$

б) множества $\left\{S\left(K, y^{*}, \alpha\right), \alpha>0\right\}$ образуют в множестве $K$ базу окрестностей точки $y_{*}$ в нормированной топологии.

В этом случае элемент $y^{*} \in Y^{\prime}$ назьвается строго выставляющим точку $y_{*}$.

Из а), б) следует, что если $y_{*}-$ строго выставленная точка, то $\left\langle y_{*}, y^{*}\right\rangle<\left\langle y, y^{*}\right\rangle$, $y \in K, y \neq y_{*}$.

Если diam означает диаметр множества в нормированной топологии, то условие б) эквивалентно условию

$$
\lim _{\alpha \rightarrow 0+} \operatorname{diam} S\left(K, y^{*}, \alpha\right)=0 .
$$

Другим эквивалентным условием является следующее: если $y_{n} \in K, n \geqslant 1$, и $\lim _{n \rightarrow \infty}\left\langle y_{n}-y_{*}, y^{*}\right\rangle=0$, то $\lim _{n \rightarrow \infty}\left\|y_{n}-y_{*}\right\|=0$.

Из определения строго выставленной точки множества следует, что она является экстремальной (крайней) точкой множества.

В дальнейшем через strexp $K$ мы будем обозначать совокупность всех строго выставленных точек множества $K$.

Пусть $(T, \Sigma, \mu)$ - измеримое пространство с неотрицательной полной $\sigma$-конечной мерой $\mu$ и $\sigma$-алгеброй $\Sigma \mu$-измеримых множеств, $(X,\|\cdot\|)$ - сепарабельное банахово пространство, $2^{X}$ - совокупность всех подмножеств из $X$, включая пустое множество, и $2_{0}^{X}-$ совокупность всех непустых подмножеств из $X$.

Обозначим через $L_{p}(T, X), 1 \leqslant p<\infty$, банахово пространство классов эквивалентности инрегрируемых по Бохнеру функций $f: T \rightarrow X$ с нормой

$$
\|f\|_{p}=\left(\int_{T}\|f(t)\|^{p} d \mu\right)^{1 / p}
$$

и через $L_{p}^{\prime}(T, X)$ пространство, топологически сопряженное к $L_{p}(T, X)$. Известно, что пространство $L_{p}^{\prime}(T, X)$ отождествимо с пространством $L_{q}\left(T, X_{\sigma}^{\prime}\right), 1 / p+1 / q=1$, скалярно измеримых функций $f^{\prime}: T \rightarrow X_{\sigma}^{\prime}[6]$, для которых функция $t \rightarrow\left\|f^{\prime}(t)\right\|$ является элементом пространства $L_{q}(T, \mathbb{R})$. Каноническая билинейная форма, устанавливающая двойственность между $L_{p}(T, X)$ и $L_{q}\left(T, X^{\prime}\right)$, имеет вид

$$
\left\langle f, f^{\prime}\right\rangle=\int_{T}\left\langle f(t), f^{\prime}(t)\right\rangle d \mu .
$$


Множество $A \subset L_{p}(T, X)$ называется разложимылм, если для любых $f, \varphi \in A$ и для любого $E \in \Sigma$ элемент $f \cdot \chi_{E}+\varphi \cdot \chi_{T \backslash E}$ принадлежит $A$, где $\chi_{E}$ означает характеристическую функцию множества $E$.

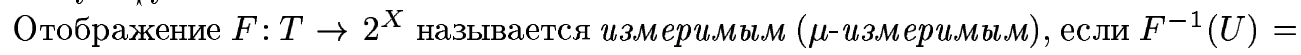
$\{t \in T \mid F(t) \cap U \neq \varnothing\} \in \Sigma$ для любого замкнутого множества $U \subset X$.

Отображение $F: T \rightarrow 2_{0}^{X}$ назьвается $p$-интегрально ограниченным, если существует элемент $\lambda \in L_{p}(T, \mathbb{R})$ такой, что

$$
\|F(t)\|=\sup \{\|x\| \mid x \in F(t)\} \leqslant \lambda(t) \text { почти всюду. }
$$

Отображение $F: T \rightarrow 2^{X}$ назьвается полунепрерывным снизу, если множество $F^{-1}(U)$ открыто для любого открытого множества $U \subset X$. Непосредственно из определения следует, что если отображение $F: T \rightarrow 2^{X}$ полунепрерывно снизу, то отображение $\bar{F}: T \rightarrow 2^{X}, \bar{F}(t)=\overline{F(t)}$, где черта означает замыкание в $X$, также полунепрерывно снизу.

Для отображения $F: T \rightarrow 2_{0}^{X}$ обозначим

$$
S^{p}(F)=\left\{f \in L_{p}(T, X) \mid f(t) \in F(t) \text { почти всюду }\right\} .
$$

Известно [7], что для замкнутого ограниченного разложимого множества $\Gamma \subset L_{p}(T, X)$, $1 \leqslant p<\infty$, существует единственное (с точностью до множества меры нуль) измеримое $p$-интегрально ограниченное отображение $F: T \rightarrow 2_{0}^{X}$ с замкнутыми значениями такое, что

$$
\Gamma=S^{p}(F)
$$

При этом если множество Г выпукло, то значениями отображения $F$ являются выпуклые множества.

Отображение $F: T \rightarrow 2_{0}^{X}$, удовлетворяющее (1.1), мы будем называть многозначным представлением множества $Г$.

Очевидно, что если $F: T \rightarrow 2_{0}^{X}$ измеримое $p$-интегрально ограниченное отображение с замкнутыми значениями, то $S^{p}(F)$ является замкнутым ограниченным разложимым множеством в пространстве $L_{p}(T, X), 1 \leqslant p<\infty$.

2. Характеризация строго выставленных точек. Всюду в дальнейшем, если не оговорено противное, $(T, \Sigma, \mu)$ - отделимое локально компактное счетное в бесконечности пространство с неотрицательной мерой Радона $\mu$, которая, как известно, является полной, а $X$ - сепарабельное банахово пространство.

Tеорема 2.1. Пусть $\Gamma \subset L_{p}(T, X), 1 \leqslant p<\infty$, - замкнутое выпуклое ограниченное разлохимое мнохсество и $F: T \rightarrow 2_{0}^{X}$ - его многозначное представление. Точка $f_{*} \in \Gamma$ является строго выставленной тогда и только тогда, когда $f(t)$ является строго выставленной точкой мнохсества $F(t)$ при почти всех $t \in T$.

ДокАЗАТЕЛЬСтво. Необходимость. Пусть $f_{*}$ - строго выставленная точка множества $\Gamma$ и $f^{*} \in L_{q}\left(T, X_{\sigma}^{\prime}\right), 1 / p+1 / q=1$, - элемент, строго выставляющий точку $f_{*}$. Согласно (1.1) $f_{*}(t) \in F(t)$ почти всюду на $T$. В соответствии с определением строго выставленной точки

$$
\left\langle f_{*}, f^{*}\right\rangle=\sup _{f \in \Gamma} \int_{T}\left\langle f(t), f^{*}(t)\right\rangle d \mu .
$$


Воспользовавшись теоремой 2.2 [7], справедливой для разложимых множеств, получаем

$$
\sup _{f \in \Gamma} \int_{T}\left\langle f(t), f^{*}(t)\right\rangle d \mu=\int_{T} \sup _{x \in F(t)}\left\langle x, f^{*}(t)\right\rangle d \mu .
$$

Из (2.1), (2.2) следует равенство

$$
\int_{T}\left\langle f_{*}(t), f^{*}(t)\right\rangle d \mu=\int_{T} \sup _{x \in F(t)}\left\langle x, f^{*}(t)\right\rangle d \mu .
$$

Так как $f_{*}(t) \in F(t)$ почти всюду, то

$$
\left\langle f_{*}(t), f^{*}(t)\right\rangle \leqslant \sup _{x \in F(t)}\left\langle x, f^{*}(t)\right\rangle .
$$

Поэтому из (2.3), (2.4) вытекает

$$
\left\langle f_{*}(t), f^{*}(t)\right\rangle=\sup _{x \in F(t)}\left\langle x, f^{*}(t)\right\rangle \quad \text { почти всюду. }
$$

Пусть $s(t)=\sup \left\{\left\langle x, f^{*}(t)\right\rangle \mid x \in F(t)\right\}$ и $\lambda \in L_{1}(T, R), \lambda(t)>0, t \in T, \int_{T} \lambda(t) d t=1$. Положим

$$
F_{n}(t)=\left\{x \in F(t) \mid s(t)-\frac{1}{n} \lambda(t) \leqslant\left\langle x, f^{*}(t)\right\rangle\right\} .
$$

Так как функция $t \rightarrow s(t)$ измерима, а функция $(x, t) \rightarrow\left\langle x, f^{*}(t)\right\rangle$ типа Каратеодори, то из $\left[8\right.$, теорема 6.4] и $\left[7\right.$, теорема 1.0] вытекает, что $F_{n}: T \rightarrow 2_{0}^{X}$ является измеримым отображением с замкнутыми выпукльми значениями.

Пусть

$$
\Gamma_{n}=\left\{f \in \Gamma \mid\left\langle f_{*}, f^{*}\right\rangle-\frac{1}{n} \leqslant\left\langle f, f^{*}\right\rangle\right\} .
$$

В соответствии с $(2.2),(2.5),(2.6)$ для любого $f \in S^{p}\left(F_{n}\right)$

$$
\sup _{\varphi \in \Gamma}\left\langle\varphi, f^{*}\right\rangle-\frac{1}{n} \leqslant\left\langle f, f^{*}\right\rangle .
$$

Поэтому $f_{*} \in S^{p}\left(F_{n}\right) \subset \Gamma_{n}$. Так как $f_{*}-$ строго выставленная точка множества $\Gamma$, то $\operatorname{diam} \Gamma_{n} \rightarrow 0$ при $n \rightarrow \infty$. Поэтому $\operatorname{diam} S^{p}\left(F_{n}\right) \rightarrow 0$ при $n \rightarrow \infty$. Поскольку $S^{p}\left(F_{n}\right)-$ замкнутое ограниченное разложимое множество, то в соответствии с [7, теорема 2.2]

$$
\sup _{f, \varphi \in S^{p}\left(F_{n}\right)}\|f-\varphi\|_{p}^{p}=\int_{T}\left\{\sup _{x, y \in F_{n}(t)}\|x-y\|^{p}\right\} d \mu .
$$

Поэтому

$$
\operatorname{diam} S^{p}\left(F_{n}\right)=\left(\int_{T}\left(\operatorname{diam} F_{n}(t)\right)^{p} d \mu\right)^{1 / p}
$$

Tak как

$$
\lim _{n \rightarrow \infty} \operatorname{diam} S^{p}\left(F_{n}\right)=0,
$$

то существует подпоследовательность $n_{k}, k \geqslant 1$, последовательности $n \geqslant 1$ такая, что почти всюду

$$
\lim _{k \rightarrow \infty} \operatorname{diam} F_{n_{k}}(t)=0
$$


Из последнего равенства и (2.5) следует, что $f_{*}(t)$ является строго выставленной точкой $F(t)$ при почти всех $t \in T$, а элемент $f^{*}(t) \in X^{\prime}$ строго выставляет точку $f_{*}(t)$. Необходимость доказана.

Достаточность. Пусть $f_{*} \in \Gamma$ и $f_{*}(t)$ является строго выставленной точкой $F(t)$ при почти каждом $t \in T$. Обозначим через $x_{*}^{\prime}(t)$ элемент из $X^{\prime}$, который строго выставляет точку $f_{*}(t)$. Пусть $B_{\sigma}^{\prime}-$ единичньй замкнутьй шар из $X^{\prime}$ со слабой $\sigma\left(X^{\prime}, X\right)$ топологией, которьй является метризуемьм компактом. В дальнейшем $B_{\sigma}^{\prime}$ мы рассматриваем как самостоятельное метрическое компактное пространство. Не нарушая общности, можно считать, что $x_{*}^{\prime}(t) \in B_{\sigma}^{\prime}$.

Из [7, теорема 1.0] следует, что существует счетное семейство измеримых селекторов $g_{n}(t), n \geqslant 1$, отображения $F(t)$ такое, что

$$
F(t)=\overline{\bigcup_{n=1}^{\infty} g_{n}(t)}, \quad t \in T,
$$

где черта означает замыкание в $X$.

Воспользовавшись определением измеримых функций из $T$ в $X$, получаем, что для любого компакта $\mathscr{T} \subset T$, любого $\varepsilon>0$ существует компактное множество $\mathscr{T}_{\varepsilon} \subset \mathscr{T}$, $\mu\left(\mathscr{T} \backslash \mathscr{T}_{\varepsilon}\right) \leqslant \varepsilon$, на котором функции $f_{*}(t), g_{n}(t), n \geqslant 1$, непрерывны и $f_{*}(t)$ является строго выставленной точкой $F(t)$ при каждом $t \in \mathscr{T}_{\varepsilon}$. Зафиксируем $\mathscr{T}_{\varepsilon}$. Тогда из $(2.7)$ следует, что $F(t)$ является полунепрерьвной снизу на $\mathscr{T}_{\varepsilon}$ многозначной функцией с непустыми замкнутыми вьпуклыми значениями.

Пусть $r\left(t, x^{\prime}\right)=\sup \left\{\left\langle y, x^{\prime}\right\rangle \mid y \in F(t)\right\}, t \in \mathscr{T}_{\varepsilon}, x^{\prime} \in B_{\sigma}^{\prime}$. Очевидно, что

$$
r\left(t, x^{\prime}\right)=\sup _{n \geqslant 1}\left\{\left\langle g_{n}(t), x^{\prime}\right\rangle\right\} .
$$

Поэтому функция $\left(t, x^{\prime}\right) \rightarrow r\left(t, x^{\prime}\right)$ полунепрерывна снизу на $\mathscr{T}_{\varepsilon} \times B_{\sigma}^{\prime}$.

Рассмотрим функции

$$
r_{n}\left(t, x^{\prime}\right)=\sup _{1 \leqslant k \leqslant n}\left\{\left\langle g_{k}(t), x^{\prime}\right\rangle\right\}, \quad t \in \mathscr{T}_{\varepsilon}, \quad x^{\prime} \in B_{\sigma}^{\prime} .
$$

Так как каждая из функций $\left(t, x^{\prime}\right) \rightarrow\left\langle g_{k}(t), x^{\prime}\right\rangle$ непрерьвна на $\mathscr{T}_{\varepsilon} \times B_{\sigma}^{\prime}$, то $r_{n}\left(t, x^{\prime}\right)$, $n \geqslant 1,-$ неубывающая последовательность непрерывных функций, поточечно сходящаяся к $r\left(t, x^{\prime}\right)$. Пусть

$$
\begin{aligned}
& F_{m}\left(t, x^{\prime}\right)=\left\{x \in F(t) \mid\left\langle x, x^{\prime}\right\rangle \geqslant r\left(t, x^{\prime}\right)-\frac{1}{m}\right\}, \\
& F_{m}^{n}\left(t, x^{\prime}\right)=\left\{x \in F(t) \mid\left\langle x, x^{\prime}\right\rangle \geqslant r_{n}\left(t, x^{\prime}\right)-\frac{1}{m}\right\} .
\end{aligned}
$$

Ясно, что $F_{m}^{n}\left(t, x^{\prime}\right), n \geqslant 1,-$ убьвающая по включению последовательность непустых выпуклых замкнутых множеств и $F_{m}\left(t, x^{\prime}\right)=\bigcap_{n=1}^{\infty} F_{m}^{n}\left(t, x^{\prime}\right)$.

Покажем, что многозначное отображение $\left(t, x^{\prime}\right) \rightarrow F_{m}^{n}\left(t, x^{\prime}\right)$ полунепрерывно снизу на $\mathscr{T}_{\varepsilon} \times B_{\sigma}^{\prime}$. Для этого достаточно показать, что многозначное отображение $\Phi_{m}^{n}\left(t, x^{\prime}\right)$, задаваемое формулой

$$
\Phi_{m}^{n}\left(t, x^{\prime}\right)=\left\{x \in F(t) \mid\left\langle x, x^{\prime}\right\rangle>r_{n}\left(t, x^{\prime}\right)-\frac{1}{m}\right\}
$$


полунепрерьвно снизу, поскольку $F_{m}^{n}\left(t, x^{\prime}\right)=\overline{\Phi_{m}^{n}\left(t, x^{\prime}\right)}$.

Пусть $U \subset X$ - открытое множество и $t_{0} \in \mathscr{T}_{\varepsilon}, x_{0}^{\prime} \in B_{\sigma}^{\prime}, x_{0} \in \Phi_{m}^{n}\left(t_{0}, x_{0}^{\prime}\right) \cap U$. Тогда $\left\langle x, x_{0}^{\prime}\right\rangle-r_{n}\left(t_{0}, x_{0}^{\prime}\right)+1 / m>0$. Так как функция $\left(x, t, x^{\prime}\right) \rightarrow\left\langle x, x^{\prime}\right\rangle-r_{n}\left(t, x^{\prime}\right)+1 / m$ непрерьвна на $X \times \mathscr{T}_{\varepsilon} \times B_{\sigma}^{\prime}$, то существуют окрестности $O\left(x_{0}\right), V\left(t_{0}\right), W\left(x_{0}^{\prime}\right)$ точек $x_{0}, t_{0}, x_{0}^{\prime}$ такие, что $\left\langle x, x^{\prime}\right\rangle-r_{n}\left(t, x^{\prime}\right)+1 / m>0$ для всех $x \in O\left(x_{0}\right), t \in V\left(t_{0}\right), x^{\prime} \in W\left(x_{0}^{\prime}\right)$. Из паракомпактности пространства $\mathscr{T}_{\varepsilon} \times B_{\sigma}^{\prime}[9]$ и теоремы Майкла [10] следует, что существует непрерьвньй селектор $x(t)$ отображения $F(t)$ такой, что $x\left(t_{0}\right)=x_{0}$. Поэтому найдется окрестность $V_{1}\left(t_{0}\right) \subset V\left(t_{0}\right)$ точки $t_{0}$ такая, что для всех $t \in V_{1}\left(t_{0}\right), x^{\prime} \in W\left(x_{0}^{\prime}\right)$ будут выполняться соотношения

$$
x(t) \in F(t) \cap U, \quad\left\langle x(t), x^{\prime}\right\rangle-r_{n}\left(t, x^{\prime}\right)+\frac{1}{m}>0 .
$$

Но это означает, что $x(t) \in \Phi_{n}^{m}\left(t, x^{\prime}\right) \cap F_{m}(t) \cap U=\Phi_{m}^{n}(t, x) \cap U$ для всех $t \in V_{1}\left(t_{0}\right)$, $x^{\prime} \in W\left(x_{0}^{\prime}\right)$. Следовательно, отображение $\left(t, x^{\prime}\right) \rightarrow \Phi_{m}^{n}\left(t, x^{\prime}\right)$ полунепрерьвно снизу в точке $\left(t_{0}, x_{0}^{\prime}\right)$. Таковым будет и отображение $\left(t, x^{\prime}\right) \rightarrow F_{m}^{n}\left(t, x^{\prime}\right)$.

Воспользовавшись теоремой 1.0 из [7], получаем, что существует счетное семейство $q_{k}^{n}\left(t, x^{\prime}\right), k \geqslant 1$, борелевских селекторов отображения $F_{m}^{n}\left(t, x^{\prime}\right)$ такое, что

$$
F_{m}^{n}\left(t, x^{\prime}\right)=\overline{\bigcup_{k=1}^{\infty} q_{k}^{n}\left(t, x^{\prime}\right),} \quad t \in \mathscr{T}_{\varepsilon}, \quad x^{\prime} \in B_{\sigma}^{\prime}
$$

Тогда функция

$$
\operatorname{diam} F_{m}^{n}\left(t, x^{\prime}\right)=\sup \left\{\left\|q_{k}^{n}\left(t, x^{\prime}\right)-q_{l}^{n}\left(t, x^{\prime}\right)\right\|, k \geqslant 1, l \geqslant 1\right\}
$$

является борелевской на $\mathscr{T}_{\varepsilon} \times B_{\sigma}^{\prime}$.

Рассмотрим множество

$$
V_{m, k}^{n}=\left\{\left(t, x^{\prime}\right) \in \mathscr{T}_{\varepsilon} \times B_{\sigma}^{\prime} \mid \operatorname{diam} F_{m}^{n}(t, x) \leqslant \frac{1}{k}\right\}
$$

которое является борелевским в $\mathscr{T}_{\varepsilon} \times B_{\sigma}^{\prime}$. Поэтому множество

$$
V=\bigcap_{k=1}^{\infty} \bigcup_{m=1}^{\infty} \bigcup_{n=1}^{\infty} V_{m, k}^{n}
$$

борелевское в $\mathscr{T}_{\varepsilon} \times B_{\sigma}^{\prime}$.

Мы утверждаем, что точка $\left(t, x^{\prime}\right) \in V$ тогда и только тогда, когда $\operatorname{diam} F_{m}\left(t, x^{\prime}\right) \rightarrow 0$ при $m \rightarrow \infty$.

В самом деле, пусть $\left(t, x^{\prime}\right) \in V$. Тогда при фиксированном $k \geqslant 1$ найдутся $m(k), n(k)$ такие, что $\left(t, x^{\prime}\right) \in V_{m(k), k}^{n(k)}$. Но это означает, что $\operatorname{diam} F_{m(k)}^{n(k)}\left(t, x^{\prime}\right) \leqslant 1 / k$. Так как $F_{m}\left(t, x^{\prime}\right) \subset F_{m(k)}^{n(k)}\left(t, x^{\prime}\right)$ при $m \geqslant m(k)$, то $\operatorname{diam} F_{m}\left(t, x^{\prime}\right) \rightarrow 0$ при $m \rightarrow \infty$.

Наоборот, пусть $\operatorname{diam} F_{m}\left(t, x^{\prime}\right) \rightarrow 0$ при $m \rightarrow \infty$. Тогда для любого $k \geqslant 1$ найдется $m(k)$ такое, что $\operatorname{diam} F_{m}\left(t, x^{\prime}\right) \leqslant 1 / k$ при $m \geqslant m(k)$. Для $m(k)$ найдется $n(k)$ такое, что

$$
r_{n(k)}\left(t, x^{\prime}\right)-\frac{2}{m(k)} \geqslant r\left(t, x^{\prime}\right)-\frac{1}{m(k)} .
$$


Поэтому $F_{2 m(k)}^{n(k)} \subset F_{m(k)}\left(t, x^{\prime}\right)$. Следовательно, $\left(t, x^{\prime}\right) \in V_{2 m(k), k}^{n(k)}$. Отсюда вытекает, что $\left(t, x^{\prime}\right) \in \bigcup_{m=1}^{\infty} \bigcup_{n=1}^{\infty} V_{m, k}^{n}$ для любого $k \geqslant 1$. Поэтому $\left(t, x^{\prime}\right) \in V$.

Рассмотрим множество

$$
W=\left\{\left(t, x^{\prime}\right) \in \mathscr{T}_{\varepsilon} \times B_{\sigma}^{\prime} \mid\left\langle f_{*}(t), x^{\prime}\right\rangle=r\left(t, x^{\prime}\right)\right\}
$$

которое очевидно совпадает с множеством

$$
\left\{\left(t, x^{\prime}\right) \in \mathscr{T}_{\varepsilon} \times B_{\sigma}^{\prime} \mid r\left(t, x^{\prime}\right)-\left\langle f_{*}(t), x^{\prime}\right\rangle \leqslant 0\right\} .
$$

Так как функция $\left(t, x^{\prime}\right) \rightarrow r\left(t, x^{\prime}\right)-\left\langle f_{*}(t), x^{\prime}\right\rangle$ полунепрерьвна снизу, то множество $W$ замкнуто. Поэтому множество $V \cap W \subset \mathscr{T}_{\varepsilon} \times B_{\sigma}^{\prime}$ борелевское.

Из определения множества $V \cap W$ следует, что $\left(t, x^{\prime}\right) \in V \cap W$ тогда и только тогда, когда элемент $x^{\prime} \in X^{\prime}$ строго выставляет точку $f_{*}(t)$. Так как $\left(t, x_{*}^{\prime}(t)\right) \in V \cap W$, $t \in \mathscr{T}_{\varepsilon}$, где $x_{*}^{\prime}(t) \in X^{\prime}$ - элемент, строго выставляющий точку $f_{*}(t)$, то на $\mathscr{T}_{\varepsilon}$ будет определено отображение $\Phi: \mathscr{T}_{\varepsilon} \rightarrow 2_{0}^{B_{\sigma}^{\prime}}$ с непустыми значениями, графиком которого является борелевское множество $V \cap W$.

Воспользовавшись [11, следствие 2$]$, получаем, что существует селектор $f^{*}: \mathscr{T}_{\varepsilon} \rightarrow B_{\sigma}^{\prime}$ отображения $\Phi(t)$ такой, что для любого открытого множества $U \subset B_{\sigma}^{\prime}$ множество $\left\{t \in \mathscr{T}_{\varepsilon} \mid f^{*}(t) \in U\right\}$ является аналитическим. Согласно [12, теорема 2.2.12] любое аналитическое множество из $\mathscr{T}_{\varepsilon}$ является $\mu$-измеримым. Поэтому $f^{*}(t)$ является слабо измеримой функцией из $\mathscr{T}_{\varepsilon}$ в $X_{\sigma}^{\prime}[6]$ и, следовательно, скалярно измеримой. Более того, элемент $f^{*}(t) \in X_{\sigma}^{\prime}$ строго выставляет точку $f_{*}(t) \in F(t), t \in \mathscr{T}_{\varepsilon}$.

Так как $T$ - локально компактное счетное в бесконечности пространство, то существует возрастающая по включению последовательность компактов $T_{n} \subset T, n \geqslant 1$, такая, что $T=\bigcup_{n=1}^{\infty} T_{n}$. На основании доказанного вьше существует возрастающая по включению последовательность $\mathscr{T}_{n} \subset T_{n}, n \geqslant 1$, компактов с $\mu\left(T_{n} \backslash \mathscr{T}_{n}\right) \leqslant 1 / n, n \geqslant 1$, и последовательность $f_{n}^{*}: \mathscr{T}_{n} \rightarrow X_{\sigma}^{\prime}$ скалярно измеримых функций таких, что $f_{n}^{*}(t)$ строго выставляет точку $f_{*}(t) \in F(t)$ при $t \in \mathscr{T}_{n}$.

Рассмотрим функцию $\varphi^{\prime}: T \rightarrow X_{\sigma}^{\prime}$, определенную по правилу $\varphi^{\prime}(t)=f_{1}^{*}(t), t \in \mathscr{T}_{1}$, $\varphi^{\prime}(t)=f_{n}^{*}(t), t \in \mathscr{T}_{n} \backslash \mathscr{T}_{n-1}, n \geqslant 2, \varphi^{\prime}(t)=0, t \in T \backslash \bigcup_{n=1}^{\infty} \mathscr{T}_{n}$. Taк как $\mu\left(T \backslash \bigcup_{n=1}^{\infty} \mathscr{T}_{n}\right)$ $=0$, то функция $\varphi^{\prime}: T \rightarrow X_{\sigma}^{\prime}$ скалярно измерима, $\varphi^{\prime}(t)$ строго выставляет точку $f_{*}(t)$ при почти каждом $t \in T$ и $\left\|\varphi^{\prime}(t)\right\| \leqslant 1$.

Пусть $\lambda \in L_{q}(T, R), \lambda(t)>0, t \in T$. Тогда функция $f^{*}(t)=\lambda(t) \cdot \varphi^{\prime}(t)$ является элементом пространства $L_{q}\left(T, X_{\sigma}^{\prime}\right)$ и $f^{*}(t)$ при почти каждом $t \in T$ строго выставляет точку $f_{*}(t) \in F(t)$. Покажем, что $f_{*} \in \Gamma$ является строго выставленной точкой множества $\Gamma$, а элемент $f^{*} \in L_{q}\left(T, X_{\sigma}^{\prime}\right)$ строго выставляет $f_{*}$.

С помощью тех же рассуждений, которые использовались при доказательстве необходимости, получаем

$$
\left\langle f_{*}, f^{*}\right\rangle=\sup \left\{\left\langle f_{*}, \varphi\right\rangle \mid \varphi \in \Gamma\right\} .
$$

Пусть последовательность $f_{n} \in \Gamma, n \geqslant 1$, такова, что

$$
\lim _{n \rightarrow \infty}\left\langle f_{n}-f_{*}, f^{*}\right\rangle=0
$$

Наша цель - доказать, что

$$
\lim _{n \rightarrow \infty}\left\|f_{n}-f_{*}\right\|_{p}=0
$$


Так как $f_{n}(t) \in F(t)$ почти всюду, то

$$
\left\langle f_{n}(t), f^{*}(t)\right\rangle \leqslant\left\langle f_{*}(t), f^{*}(t)\right\rangle=\sup \left\{\left\langle y, f^{*}(t)\right\rangle \mid y \in F(t)\right\} \text {. }
$$

Поэтому

$$
\left\langle f_{*}-f_{n}, f^{*}\right\rangle=\int_{T}\left|\left\langle f_{*}(t)-f_{n}(t), f^{*}(t)\right\rangle\right| d \mu \rightarrow 0, \quad n \rightarrow \infty .
$$

Следовательно, существует подпоследовательность $f_{n_{k}}, k \geqslant 1$, последовательности $f_{n}$, $n \geqslant 1$, такая, что $\left\langle f_{n_{k}}(t), f^{*}(t)\right\rangle \rightarrow\left\langle f_{*}, f^{*}(t)\right\rangle$ почти всюду при $k \rightarrow \infty$. Но это означает, что $f_{n_{k}}(t) \rightarrow f^{*}(t)$ почти всюду при $k \rightarrow \infty$. Теперь из $p$-интегральной ограниченности отображения $F(t)$ и теоремы Лебега об ограниченной сходимости следует, что последовательность $f_{n_{k}} \in \Gamma, k \geqslant 1$, сходится к $f_{*}$ в $L_{p}(T, X)$. Воспользовавшись хорошо известными аргументами, мы получаем, что и сама последовательность $f_{n}, n \geqslant 1$, сходится к $f_{*}$ в $L_{p}(T, X)$. Принимая во внимание $(2.8)$, мы заключаем, что $f_{*}$ является строго выставленной точкой множества $\Gamma$, а элемент $f^{*} \in L_{q}\left(T, X^{\prime}\right)$ строго выставляет $f_{*}$. Достаточность доказана.

СлЕДСТВИЕ 2.1. Пусть $Г \subset L_{p}(T, X), 1 \leqslant p<\infty,-$ замкнутое выпуклое ограниченное разложимое мнохество. Тогда strexp Г является разложимым множеством.

ЗАмЕчАниЕ 2.1. При доказательстве необходимости в теореме 2.1 мы не использовали топологические свойства пространства $T$, а использовали только теоремы $1.0,2.2$ из [7] и теорему 6.4 из [8], которые справедливы для произвольного измеримого пространства с полной неотрицательной $\sigma$-конечной мерой. Поэтому необходимость утверждения теоремы 2.1 справедлива для любого измеримого пространства $(T, \Sigma, \mu)$ с полной неотрицательной $\sigma$-конечной мерой $\mu$.

Воспользовавшись теоремой 2.1, дадим необходимые условия, характеризующие строго выставленные точки единичного шара из $L_{p}(T, X), 1 \leqslant p<\infty$.

В дальнейшем $(T, \Sigma, \mu)$ - измеримое пространство с полной неотрицательной $\sigma$-конечной мерой $\mu, B_{p}$ и $B(X)$ - единичные замкнутые шары из пространств $L_{p}(T, X)$, $1 \leqslant p<\infty$, и $X$ соответственно. Если $f \in L_{p}(T, X)$, то $\operatorname{supp} f=\{t \in T \mid\|f(t)\| \neq 0\}$.

Лемма 2.1. Пусть $f_{*} \in \operatorname{strexp} B_{p}, 1 \leqslant p<\infty, u f^{*} \in L_{q}\left(T, X_{\sigma}^{\prime}\right), 1 / p+1 / q=1,-$ әлемент, строго выставляющий точку $f_{*}$. Тогда $\left\|f_{*}\right\|_{p}=1 u$

a) $f_{*}(t) /\left\|f_{*}(t)\right\| \in \operatorname{strexp} B(X)$ nри почти всеx $t \in \operatorname{supp} f_{*}$;

б) әлемент $\left\|f_{*}(t)\right\| \cdot f^{*}(t) \in X^{\prime}$ при почти всех $t \in \operatorname{supp} f_{*}$ строго выставляет mочку $f_{*}(t) /\left\|f_{*}(t)\right\|$.

ДоказАтельство. Так как $f_{*} \in \operatorname{ext} B_{p}$, то $\left\|f_{*}\right\|_{p}=1$. Рассмотрим многозначное отображение $F: T \rightarrow 2_{0}^{X}$, определенное по правилу $F(t)=\left\|f_{*}(t)\right\| \cdot B(X)$. Тогда $F(t)$ является измеримьм многозначным отображением с замкнутьми вьпуклыми значениями. Поэтому $S^{p}(F)$ является замкнутым выпуклым ограниченным разложимым множеством и $f_{*} \in S^{p}(F) \subset B_{p}$. Следовательно, $f_{*} \in \operatorname{strexp} S^{p}(F)$.

Тогда в соответствии с утверждением теоремы 2.1 и замечанием 2.1

$$
f_{*}(t) \in \operatorname{strexp} F(t) \quad \text { почти всюду на } T \text {. }
$$

Теперь утверждения а) и б) леммы с очевидностью вытекают из (2.9). 
Подобная характеризация строго выставленных точек множества $S^{1}(F)$ измеримого интегрально ограниченного отображения $F: T \rightarrow X$ с замкнутыми выпуклыми значениями, где $T=[0,1]$ - отрезок числовой прямой с мерой Лебега, была дана в недавно вышедшей работе [4], но в ней использовались другие аргументы.

Необходимые и достаточные условия для строго выставленных точек множества $B_{p}$, $1<p<\infty$, в терминах утверждения леммы 2.1 для произвольного банахова пространства $X$ были даны в работах [10]-[12].

Утверждения леммы 2.1 дополняют результаты работ [13]-[15] в отношении необходимых условий для случая $p=1$.

В заключение отметим, что в работе [2] было введено понятие разложимой оболочки множества strexp $Г$ как наименњшего по включению разложимого множества, содержащего множество strexp $\Gamma$. В соответствии со следствием 2.1 разложимая оболочка множества strexp Г совпадает с множеством strexp $Г$. Этот факт позволяет упростить доказательство ряда результатов из [2], [16].

\section{СПИСОК ЦИТИРОВАННОЙ ЛИТЕРАТУРЫ}

[1] Castaing C., Valadier M. Convex Analysis and Measurable Multifunctions. Lecture Notes in Math. V. 580. Berlin: Springer, 1977.

[2] Tolstonogov A.A., Tolstonogov D.A. $L_{p}$-continuous extreme selectors of multifunctions with decomposable values: existence theorems // Set-Valued Anal. 1996. V. 4. P. 173-203.

[3] Benabdellah H. Denting points in Bochner Banach ideal spaces $X(E)$ // J. Convex Analysis. 1999. V. 6. P. 183-194.

[4] Суслов С.И. Характеризация сильно выступающих точек в $L_{1}(T, X) / /$ Сиб. матем. ж. 2000. Т. 41. № 3. С. 692-695.

[5] Bourgin R. D. Geometric Aspects of Convex Sets with the Radon-Nikodym Property. Lecture Notes in Math. V. 993. Berlin: Springer, 1983.

[6] Эдвардс Р. Функциональный анализ. Теория и приложения. М.: Мир, 1969.

[7] Hiai F., Umegaki H. Integrals, conditional expectations, and martingales of multivalued functions // J. Multivariate Anal. 1977. V. 7. P. 149-182.

[8] Himmelberg C. J. Measurable relations // Fund. Math. 1975. V. 87. P. 53-72.

[9] Бурбаки Н. Общая топология. Основные структуры. М.: Наука, 1968.

[10] Michael E. Continuous selections, I // Ann. Math. 1956. V. 63. P. 361-382.

[11] Левин В. Л. Измеримые сечения многозначных отображений и проекции измеримых множеств // Функцион. анализ и его прилож. 1978. Т. 12. № 2. С. 40-45.

[12] Федерер Г. Геометрическая теория меры. М.: Наука, 1987.

[13] Greim P. Strongly exposed points in Bochner $L^{p}$-spaces // Proc. Amer. Math. Soc. 1983. V. 88. P. $81-84$.

[14] Zhibao Hu, Bor-Luh Lin. Strongly exposed points in Lebesgue-Bochner function spaces // Proc. Amer. Math. Soc. 1994. V. 120. P. 1159-1165.

[15] Hensgen W. Exposed points in Lebesgue-Bochner and Hardy-Bochner Spaces // J. Math. Anal. Appl. 1996. V. 198. P. 780-795.

[16] Tolstonogov A. A., Tolstonogov D.A. $L_{p}$-continuous extreme selectors of multifunctions with decomposable values: relaxation theorems // Set-Valued Anal. 1996. V. 4. P. 237-269.

Институт динамики систем и теории управления СО РАН

E-mail: aatiol@icc.ru.

Поступило

17.08 .2000

Исправленный вариант

30.11 .2000 